Numerical Solutions for Gyrotactic Bioconvection of Dusty Nanofluid along a Vertical Isothermal Surface

N. Begum, S. Siddiqa, M. Sulaiman, S. Islam, M. A. Hossain, R. S. R. Gorla 


\title{
Numerical Solutions for Gyrotactic Bioconvection of Dusty Nanofluid along a Vertical Isothermal Surface
}

\author{
Naheed Begum ${ }^{b}$, Sadia Siddiqa ${ }^{*, 1}$, M. Sulaiman ${ }^{* \ddagger}$, S. Islam ${ }^{\ddagger}$, M. A. Hossain ${ }^{\S}$, R. S. R. \\ Gorla $^{\dagger}$ \\ * Department of Mathematics, COMSATS Institute of Information Technology, Kamra \\ Road, Attock, Pakistan \\ ${ }^{b}$ Institute of Applied Mathematics (LSIII), TU Dortmund, Vogelpothsweg 87, D-44227 \\ Dortmund, Germany \\ $\S$ UGC Professor, Department of Mathematics, University of Dhaka, Dhaka, Bangladesh \\ ${ }^{\ddagger}$ Department of Mathematics, Abdul Wali Khan University, Mardan, Khyber \\ Pakhtunkhwa, Pakistan \\ ${ }^{\dagger}$ Department of Mechanical \&3 Civil Engineering, Purdue University Northwest, Westville, \\ IN 46391
}

\section{Abstract}

The aim of present paper is to establish the detailed numerical results for bioconvection boundary-layer flow of two-phase dusty nanofluid. The dusty fluid contains gyrotactic microorganisms along an isothermally heated vertical wall. The physical mechanisms responsible for the slip velocity between the dusty fluid and nanoparticles, such as thermophoresis and Brownian motion, are included in this study. The influence of the dusty nanofluid on heat transfer and flow characteristics are investigated in this paper. The governing equations for two-phase model are non-dimensionalized and then solved numerically via twopoint finite difference method together with the tri-diagonal solver. Results are presented graphically for wall skin friction coefficient, rate of heat transfer, velocity and temperature profiles and streamlines and isotherms. To ensure the accuracy, the computational results are compared with available data and are found in good agreement. The key observation from present analysis is that the mass concentration parameter, $D_{\rho}$, extensively promotes the rate of heat transfer, $Q_{w}$, whereas, the wall skin friction coefficient, $\tau_{w}$, is reduced by loading the dust parameters in water based dusty nanofluid.

Keywords: Nanofluid, Bioconvection, Gyrotactic microorganisms, Dusty fluid, Two-phase, Isothermal surface.

\section{Introduction}

In fluid mechanics, bioconvection is a natural phenomenon that primely related with the self-propelled microorganisms's suspension. It is worthy to mention here that, bioconvection is different from typical multi-phase flows, where particles behavior is not self-propelled; they are just carried by the fluid flow. Bioconvection originates due to instability in density stratification, which is created by directional swimming of microorganisms that are heavier than their surrounding fluid (i.e., water). These self-propelled motile microorganisms tends to concentrate near the upper portion of the fluid layer, and this accumulation makes the

\footnotetext{
${ }^{1}$ Corresponding Author.

Email: saadiasiddiqa@gmail.com
} 
upper layer much denser than the lower region and ultimately produce instability, which results in generating the various flow patterns into the system (for details see Refs. [1]-[6]). Bioconvection has numerous applications in biological and bio-microsystems. In addition, another potential application of theory of bioconvection is microbial-enhanced oil recovery, where nutrients and microorganisms are injected in layers of oil-bearing for correcting the permeability variation. Besides, the property of directional motion of motile microorganisms may be used for the concentration of cells, separation of dead and living cells, purification of cultures, or separation of various sub-populations [7]-[9]. However, bioconvection systems may be classified depending upon the directional movement of various species of microorganisms, but, they usually swim in upward direction (having larger density than the base fluid). For instance, i) chemotaxis or oxytactic type of microorganisms swims upwardly due to the gradient of oxygen concentration, as they require certain amount of oxygen concentration to be active, ii) gyrotactic microorganisms are the ones whose swimming's direction is determined by making a balance in viscous and gravitational torques, and iii) geotactic microorganisms swim against the gravitational effects [10]-[11]. In addition, the concept of nanofluid bioconvection has also wide circle of applications, for instance, nanomaterial processing, automotive coolants, sterilization process of medical suspensions and polymer coating. The idea of nanofluid bioconvection was first introduced by Kuznetsov [12]-[13], and later on, due to the characteristics of nanofluids to promote the rate of heat transfer, numerous authors and analysts investigated the interaction of nanofluid with bioconvection (see Refs. [14]-[20]).

The dynamics of gas-particle flows were of great interest in the past half century because of their wide spectrum of practical and technical applications in atmospheric, engineering and physiological fields. For instance, fluidized beds, conveying of powdered materials, purification of crude oil, environmental pollutants, combustion chambers, petroleum industry are some of the worthy-mentioned applications of particulate suspensions [21]. The first experimental analysis on gas-particulate suspension flow was made by Farbar and Morley [22], and later on, Marble [23] developed the mathematical equations for dusty fluid flow systems. Singleton was the first to make the boundary layer analysis for gas-particle flows, which is important to determine the accumulation of particles in suspension and their impingement on the surface [24]. Afterwards, several studied were done to give a physical insight to such two-phase dusty flows under different physical circumstances [25]-[30]. However, it is found that the problem of two-phase dusty nanofluid with gyrotactic bioconvection along a vertical surface has not been treated in the literature. Therefore, in the view of above discussion, present study is presented to investigate the influence of small solid particles on nanofluid bioconvection flow. It is assumed that i) the gyrotactic microorganisms are self-propelled and ii) the nanoparticles movement is due to the Brownian motion and thermophoresis and they are carried by the dusty fluid. On the basis of these physical assumption, the interaction of microorganisms, dust particles and nanoparticles are expected to present an interesting problem in area of fluid dynamics. The governing set of boundary-layer equations are converted into a convenient form through coordinate transformation known as primitive variable formulations (PVF). These nonlinear and coupled equations are solved numerically by using iterative finite difference method. The computational results are presented graphically in the form of streamlines and isotherms, velocity and temperature profiles, skin friction coefficient and rate of heat transfer by varying different physical parameters. 


\section{Flow Analysis}

Consideration has been given to the bioconvection boundary-layer flow of dusty nanofluid along a vertical isothermal wall. The surface of the wall is heated with temperature, $T_{w}$ and it is assumed that $T_{w}>>T_{\infty}$, where $T_{\infty}$ is the ambient temperature of the base fluid. For detailed numerical simulations we have supposed that i) the dust particles are of uniform size and equally distributed in water based nanofluid, ii) nanoparticles does not affect the microorganism's swimming direction and velocity and iii) bioconvection is only induced in purely sluggish cell suspension. In the light of above conditions, the physical model describing the bioconvection flow can be written as (for details see Refs. [15], [23], [28]):

$$
\begin{gathered}
\frac{\partial \bar{u}}{\partial \bar{x}}+\frac{\partial \bar{v}}{\partial \bar{y}}=0 \\
\rho\left(\bar{u} \frac{\partial \bar{u}}{\partial \bar{x}}+\bar{v} \frac{\partial \bar{u}}{\partial \bar{y}}\right)=-\frac{\partial \bar{p}}{\partial \bar{x}}+\mu\left(\frac{\partial^{2} \bar{u}}{\partial \bar{x}^{2}}+\frac{\partial^{2} \bar{u}}{\partial \bar{y}^{2}}\right)+\left(\rho g \beta\left(1-\phi_{\infty}\right)\left(T-T_{\infty}\right)-g\left(\rho_{n p}-\rho\right)\left(\phi-\phi_{\infty}\right)\right. \\
\left.-g \gamma\left(\rho_{m}-\rho\right)\left(n-n_{\infty}\right)\right)+\frac{\rho_{p}}{\tau_{m}}\left(\bar{u}_{p}-\bar{u}\right)
\end{gathered}
$$

$$
\begin{gathered}
\rho\left(\bar{u} \frac{\partial \bar{v}}{\partial \bar{x}}+\bar{v} \frac{\partial \bar{v}}{\partial \bar{y}}\right)=-\frac{\partial \bar{p}}{\partial \bar{y}}+\mu\left(\frac{\partial^{2} \bar{v}}{\partial \bar{x}^{2}}+\frac{\partial^{2} \bar{v}}{\partial \bar{y}^{2}}\right)+\frac{\rho_{p}}{\tau_{m}}\left(\bar{v}_{p}-\bar{v}\right) \\
\bar{u} \frac{\partial T}{\partial \bar{x}}+\bar{v} \frac{\partial T}{\partial \bar{y}}=\frac{\kappa}{\rho c_{p}}\left(\frac{\partial^{2} T}{\partial \bar{x}^{2}}+\frac{\partial^{2} T}{\partial \bar{y}^{2}}\right)+\tau\left[D_{B}\left(\frac{\partial \phi}{\partial \bar{x}} \frac{\partial T}{\partial \bar{x}}+\frac{\partial \phi}{\partial \bar{y}} \frac{\partial T}{\partial \bar{y}}\right)+\frac{D_{T}}{T_{\infty}}\left(\left(\frac{\partial T}{\partial \bar{x}}\right)^{2}+\left(\frac{\partial T}{\partial \bar{y}}\right)^{2}\right)\right] \\
+\frac{\rho_{p} c_{s}}{\tau_{T} \rho c_{p}}\left(T_{p}-T\right)
\end{gathered}
$$

$$
\begin{gathered}
\bar{u} \frac{\partial \phi}{\partial \bar{x}}+\bar{v} \frac{\partial \phi}{\partial \bar{y}}=D_{B}\left(\frac{\partial^{2} \phi}{\partial \bar{x}^{2}}+\frac{\partial^{2} \phi}{\partial \bar{y}^{2}}\right)+\frac{D_{T}}{T_{\infty}}\left(\frac{\partial^{2} T}{\partial \bar{x}^{2}}+\frac{\partial}{\partial \bar{u}^{2}}+\frac{\partial}{\partial \bar{y}}(n \bar{v})+\frac{b W_{m o}}{\left(\phi_{w}-\phi_{\infty}\right)}\left[\frac{\partial}{\partial \bar{x}}\left(n \frac{\partial \phi}{\partial \bar{x}}\right)+\frac{\partial}{\partial \bar{y}}\left(n \frac{\partial \phi}{\partial \bar{y}}\right)\right]\right. \\
\frac{\partial \bar{u}_{p}}{\partial \bar{x}}+\frac{\partial \bar{v}_{p}}{\partial \bar{y}}=0 \\
\rho_{p}\left(\bar{u}_{p} \frac{\partial \bar{u}_{p}}{\partial \bar{x}}+\bar{v}_{p} \frac{\partial \bar{u}_{p}}{\partial \bar{y}}\right)=-\frac{\partial \bar{p}_{p}}{\partial \bar{x}}-\frac{\rho_{p}}{\tau_{m}}\left(\bar{u}_{p}-\bar{u}\right) \\
\rho_{p}\left(\bar{u}_{p} \frac{\partial \bar{v}_{p}}{\partial \bar{x}}+\bar{v}_{p} \frac{\partial \bar{v}_{p}}{\partial \bar{y}}\right)=-\frac{\partial \bar{p}_{p}}{\partial \bar{y}}-\frac{\rho_{p}}{\tau_{m}}\left(\bar{v}_{p}-\bar{v}\right) \\
\rho_{p} c_{s}\left(\bar{u}_{p} \frac{\partial T}{\partial \bar{x}}+\bar{v}_{p} \frac{\partial T}{\partial \bar{y}}\right)=-\frac{\rho_{p} c_{s}}{\tau_{T}}\left(T_{p}-T\right)
\end{gathered}
$$

$\bar{u} \frac{\partial n}{\partial \bar{x}}+\bar{v} \frac{\partial n}{\partial \bar{y}}+\frac{\partial}{\partial \bar{x}}(n \bar{u})+\frac{\partial}{\partial \bar{y}}(n \bar{v})+\frac{b W_{m o}}{\left(\phi_{w}-\phi_{\infty}\right)}\left[\frac{\partial}{\partial \bar{x}}\left(n \frac{\partial \phi}{\partial \bar{x}}\right)+\frac{\partial}{\partial \bar{y}}\left(n \frac{\partial \phi}{\partial \bar{y}}\right)\right]=D_{m o}\left(\frac{\partial^{2} n}{\partial \bar{x}^{2}}+\frac{\partial^{2} n}{\partial \bar{y}^{2}}\right)$

The corresponding boundary conditions are:

$$
\begin{aligned}
& \bar{u}(\bar{x}, 0)=\bar{v}(\bar{x}, 0)=T(\bar{x}, 0)-T_{w}=\phi(\bar{x}, 0)-\phi_{w}=n(\bar{x}, 0)-n_{w}=0 \\
& \bar{u}(\bar{x}, \infty)=\bar{v}(\bar{x}, \infty)=T(\bar{x}, \infty)-T_{\infty}=\phi(\bar{x}, \infty)-\phi_{\infty}=n(\bar{x}, \infty)-n_{\infty}=0
\end{aligned}
$$




$$
\begin{aligned}
& \bar{u}_{p}(\bar{x}, 0)=\bar{v}_{p}(\bar{x}, 0)=T_{p}(\bar{x}, 0)-T_{w}=0, \\
& \bar{u}_{p}(\bar{x}, \infty)=\bar{v}_{p}(\bar{x}, \infty)=T_{p}(\bar{x}, \infty)-T_{\infty}=0
\end{aligned}
$$

where, $(\bar{u}, \bar{v})$ are the components of the velocity field in $(\bar{x}, \bar{y})$ direction, $\bar{p}$ the pressure, $T$ the temperature, $\phi$ the nanoparticle concentration and $n$ the microorganisms concentration for the fluid phase. Similarly, $\left(\bar{u}_{p}, \bar{v}_{p}\right)$ are $(\bar{x}, \bar{y})$ components of velocity field, $\bar{p}_{p}$ the pressure and $T_{p}$ the temperature for the particle phase. Further, $\phi_{w}$ is the nanoparticle volume fraction, $n_{w}$ the density of microorganisms at the vertical surface, $\phi_{\infty}$ the ambient conditions at volume fraction of nanoparticles, $n_{\infty}$ the density of the microorganisms, $\beta$ the volumetric expansion coefficient of the base fluid (nanofluid), $\rho$ the density of the base fluid, $\mu$ the dynamic viscosity, $\rho_{p}$ the density of dust particles, $\rho_{n p}$ the density of the nanoparticles, $\rho_{m}$ the microorganisms density, $g$ the acceleration due to gravity force, $\kappa$ the thermal conductivity of the nanofluid, $\gamma$ the average volume of a microorganism, $c_{p}$ the specific heat at constant pressure for base fluid (particles), $c_{s}$ the specific heat at constant pressure for the particles, $\tau_{m}$ the momentum relaxation time, $\tau_{T}$ the thermal relaxation time, $D_{B}$ Brownian diffusion coefficient, $D_{T}$ thermophoretic diffusion coefficient, $b$ chemotaxis constant, $D_{m 0}$ diffusivity of microorganisms, $W_{m 0}$ the maximum cell swimming speed and $\tau=(\rho c)_{p} /(\rho c)_{f}$ the ratio of heat capacity of nanofluid to the heat capacity of the base fluid, respectively. The system can be non-dimensionalized with the help of following variables:

$$
\begin{aligned}
& x=\frac{\bar{x}}{L}, \quad y=\frac{\bar{y}}{L} G r_{L}^{1 / 4}, \quad\left(\bar{u}, \bar{u}_{p}\right)=\frac{\nu_{\infty} G r_{L}^{1 / 2}}{L}\left(u, u_{p}\right), \quad\left(\bar{v}, \bar{v}_{p}\right)=\frac{\nu_{\infty} G r_{L}^{1 / 4}}{L}\left(v, v_{p}\right), \\
& \left(\bar{p}, \bar{p}_{p}\right)=\frac{G r_{L} \rho \nu_{\infty}^{2}}{L^{2}}\left(p, p_{p}\right), \quad C=\frac{\phi-\phi_{\infty}}{\phi_{w}-\phi_{\infty}}, \quad N=\frac{n-n_{\infty}}{n_{w}-n_{\infty}}, \quad G r_{L}=\frac{g \beta\left(1-\phi_{\infty}\right)\left(T_{w}-T_{\infty}\right) L^{3}}{\nu_{\infty}^{2}}, \\
& \left(\theta, \theta_{p}\right)=\frac{\left(T, T_{p}\right)-T_{\infty}}{T_{w}-T_{\infty}}, \quad L b=\frac{\nu_{\infty}}{D_{m o}}, \quad N_{A}=\frac{D_{T}\left(T_{w}-T_{\infty}\right)}{D_{B} T_{\infty}\left(\phi_{w}-\phi_{\infty}\right)}, \quad P e=\frac{b W_{m o}}{D_{m o}}, \quad L n=\frac{\nu_{\infty}}{D_{B}}, \\
& N r=\frac{\left(\rho_{n p}-\rho\right)\left(\phi_{w}-\phi_{\infty}\right)}{\rho \beta\left(1-\phi_{\infty}\right)\left(T_{w}-T_{\infty}\right)}, \quad \operatorname{Pr}=\frac{\nu_{\infty}}{\alpha}, \quad R b=\frac{\left(\rho_{m}-\rho\right) \gamma\left(n_{w}-n_{\infty}\right)}{\rho \beta\left(1-\phi_{\infty}\right)\left(T_{w}-T_{\infty}\right)}, \quad N_{B}=\tau\left(\phi_{w}-\phi_{\infty}\right), \\
& \Omega=\frac{n_{\infty}}{\left(n_{w}-n_{\infty}\right)}, \quad \omega=\frac{c_{s}}{c_{p}}, \quad \tau_{T}=\frac{3}{2} \omega \tau_{m} \operatorname{Pr}, \quad D_{\rho}=\frac{\rho_{p}}{\rho}, \quad \alpha_{d}=\frac{L^{2}}{\tau_{m} \nu_{\infty} G r_{L}^{1 / 2}}
\end{aligned}
$$

Incorporating the dimensionless variables in Eq. (13) into the system of Eqs. (1)-(12), we get:

$$
\begin{aligned}
& \frac{\partial u}{\partial x}+\frac{\partial v}{\partial y}=0 \\
& u \frac{\partial u}{\partial x}+v \frac{\partial u}{\partial y}=-\frac{\partial p}{\partial x}+\frac{\partial^{2} u}{\partial y^{2}}+\theta-N r C-R b N+D_{\rho} \alpha_{d}\left(u_{p}-u\right) \\
& \frac{\partial p}{\partial y}=0 \\
& u \frac{\partial \theta}{\partial x}+v \frac{\partial \theta}{\partial y}=\frac{1}{\operatorname{Pr}} \frac{\partial^{2} \theta}{\partial y^{2}}+\frac{N_{B}}{L n} \frac{\partial C}{\partial y} \frac{\partial \theta}{\partial y}+\frac{N_{A} N_{B}}{L n}\left(\frac{\partial \theta}{\partial y}\right)^{2}+\frac{2}{3 \operatorname{Pr}} D_{\rho} \alpha_{d}\left(\theta_{p}-\theta\right) \\
& u \frac{\partial C}{\partial x}+v \frac{\partial C}{\partial y}=\frac{1}{L n}\left(\frac{\partial^{2} C}{\partial y^{2}}+N_{A} \frac{\partial^{2} \theta}{\partial y^{2}}\right) \\
& u \frac{\partial N}{\partial x}+v \frac{\partial N}{\partial y}+\frac{\partial}{\partial x}[(N+\Omega) u]+\frac{\partial}{\partial y}[(N+\Omega) v]+\frac{P e}{L b} \frac{\partial}{\partial y}\left[(N+\Omega) \frac{\partial C}{\partial y}\right]=\frac{1}{L b} \frac{\partial^{2} N}{\partial y^{2}}
\end{aligned}
$$




$$
\begin{gathered}
\frac{\partial u_{p}}{\partial x}+\frac{\partial v_{p}}{\partial y}=0 \\
u_{p} \frac{\partial u_{p}}{\partial x}+v_{p} \frac{\partial u_{p}}{\partial y}=-\frac{\partial p_{p}}{\partial x}-\alpha_{d}\left(u_{p}-u\right) \\
\frac{\partial p_{p}}{\partial y}=0 \\
u_{p} \frac{\partial \theta_{p}}{\partial x}+v_{p} \frac{\partial \theta_{p}}{\partial y}=-\frac{2}{3 \omega \operatorname{Pr}} \alpha_{d}\left(\theta_{p}-\theta\right)
\end{gathered}
$$

where

$L b$; the Lewis number,

$P e$; the Pèclet number,

$N r$; the buoyancy ratio parameter,

$L n$; the nanoparticle Lewis number,

$\omega$; the ratio of specific heat of the suspension,

$N_{B}$; the modified particle-density increment parameter,

$\Omega$; microorganisms concentration difference parameter,

$G r_{L}$; the Grashof number which measures the ratio of the buoyancy to viscous force,

Pr; the Prandtl number which measures the ratio of momentum diffusivity to thermal diffusivity,

$N_{A}$, the modified diffusivity ratio similar to the Soret parameter in cross diffusion phenomenon,

$\alpha_{d}$; dust parameter depending on the relaxation time of the particles and the buoyancy force,

$D_{\rho}$; mass concentration of particle phase or the ratio of density of the nanofluid to the density of the dust particles,

$R b$; the bioconvection Rayleigh number. It is worth mentioning that the bioconvection Rayleigh number $R b$ is associated with the buoyancy driven flow due to the existence of upswimming microorganisms. The concentration of microorganisms is therefore measured by the bioconvection Rayleigh number, $R b$, which by definition is always nonnegative (the zero value of $R b$ corresponds to a suspension with no microorganisms). The increase of $R b$ thus destabilizes the suspension.

The boundary conditions can be written as:

$$
\begin{gathered}
u(x, 0)=v(x, 0)=0, \quad \theta(x, 0)=C(x, 0)=N(x, 0)=1, \\
u(x, \infty)=v(x, \infty)=0, \theta(x, \infty)=0, C(x, \infty)=0, N(x, \infty)=0 \\
u_{p}(x, 0)=v_{p}(x, 0)=0, \quad \theta_{p}(x, 0)=1, \\
u_{p}(x, \infty)=v_{p}(x, \infty)=0, \theta_{p}(x, \infty)=0
\end{gathered}
$$

For the numerical solutions of the above system of Eqs. (14)-(23) along with the boundary conditions Eqs. (24)-(25), we switched into another set of equations with the help of following transformations:

$x=X, y=Y x^{\frac{1}{4}}, \quad\left(u, u_{p}\right)=x^{\frac{1}{2}}\left(U, U_{p}\right), \quad\left(v, v_{p}\right)=x^{\frac{-1}{4}}\left(V, V_{p}\right), C=C, \quad N=N, \quad \theta=\Theta$. 
Following set of equations are thus obtained:

$$
\begin{gathered}
\frac{1}{2} U+X \frac{\partial U}{\partial X}-\frac{Y}{4} \frac{\partial U}{\partial Y}+\frac{\partial V}{\partial Y}=0 \\
\frac{1}{2} U^{2}+X U \frac{\partial U}{\partial X}+\left(V-\frac{1}{4} Y U\right) \frac{\partial U}{\partial Y}=\frac{\partial^{2} U}{\partial Y^{2}}+\Theta-N r C-R b N+D_{\rho} \alpha_{d} X^{1 / 2}\left(U_{p}-U\right) \\
X U \frac{\partial \Theta}{\partial X}+\left(V-\frac{1}{4} Y U\right) \frac{\partial \Theta}{\partial Y}=\frac{1}{\operatorname{Pr}} \frac{\partial^{2} \Theta}{\partial Y^{2}}+\frac{N_{B}}{L n} \frac{\partial C}{\partial Y} \frac{\partial \Theta}{\partial Y}+\frac{N_{A} N_{B}}{L n}\left(\frac{\partial \Theta}{\partial Y}\right)^{2} \\
\quad+\frac{2}{3 \operatorname{Pr}} D_{\rho} \alpha_{d} X^{1 / 2}\left(\Theta_{p}-\Theta\right) \\
X U \frac{\partial C}{\partial X}+\left(V-\frac{1}{4} Y U\right) \frac{\partial C}{\partial Y}=\frac{1}{L n}\left(\frac{\partial^{2} C}{\partial Y^{2}}+N_{A} \frac{\partial^{2} \Theta}{\partial Y^{2}}\right) \\
+2\left(V-\frac{1}{4} Y U\right) \frac{\partial N}{\partial Y}=\frac{1}{L b}\left(\frac{\partial^{2} N}{\partial Y^{2}}-P e\left((N+\Omega) \frac{\partial^{2} C}{\partial Y^{2}}+\frac{\partial N}{\partial Y} \frac{\partial C}{\partial Y}\right)\right) \\
\frac{1}{2} U_{p}+X \frac{\partial U_{p}}{\partial X}-\frac{1}{4} Y \frac{\partial U_{p}}{\partial Y}+\frac{\partial V_{p}}{\partial Y}=0 \\
X U_{p}^{2}+X U_{p} \frac{\partial U_{p}}{\partial X}+\left(V_{p}-\frac{1}{4} Y U_{p}\right) \frac{\partial U_{p}}{\partial Y}=-\alpha_{d} X^{1 / 2}\left(U_{p}-U\right) \\
\left(V_{p}-\frac{1}{4} Y U_{p}\right) \frac{\partial \Theta_{p}}{\partial Y}=-\frac{2}{3 \omega \operatorname{Pr}} \alpha_{d} X^{1 / 2}\left(\Theta_{p}-\Theta\right)
\end{gathered}
$$

together with the boundary conditions:

$$
\begin{gathered}
U(X, 0)=V(X, 0)=0, \quad \Theta(X, 0)=C(X, 0)=N(X, 0)=1 \\
U(X, \infty)=V(X, \infty)=0, \Theta(X, \infty)=C(X, \infty)=N(X, \infty)=0 \\
U_{p}(X, 0)=V_{p}(X, 0)=0, \quad \Theta_{p}(X, 0)=1, \\
U_{p}(X, \infty)=V_{p}(X, \infty)=0, \Theta_{p}(X, \infty)=0
\end{gathered}
$$

The non-linear system of Eqs. (27)-(37) are solved with two-point implicit finite difference method. The discretization procedure and numerical scheme is carried out by considering the details given in [31]. After determining all the unknown of the system, the dimensionless expressions for the physical quantities of interest like skin friction coefficient, $\tau_{w}$ and heat transfer rate $Q_{w}$ are obtained as:

$$
\begin{gathered}
\tau_{w}=C_{f}\left(\frac{G r^{-3}}{X}\right)^{1 / 4}=\left(\frac{\partial U}{\partial Y}\right)_{Y=0} \\
Q_{w}=N u\left(\frac{G r}{X}\right)^{-1 / 4}=-\left(\frac{\partial \Theta}{\partial Y}\right)_{Y=0}
\end{gathered}
$$

The numerical results are discussed in the next section. 


\section{Results and Discussion}

In this study the effect of gyrotactic bioconvection on boundary-layer flow of two-phase dusty nanofluid are analyzed. We have performed two-dimensional simulations in order to obtain the solutions of mathematical model from the two-point implicit finite difference method. Numerical results are obtain to report the overall effectiveness of mass concentration of dust particles and nanoparticles in base fluid moving along a vertical surface. Particularly, the solutions are established for the water-based dusty nanofluid i.e., $\operatorname{Pr}=7.0, D_{\rho}=10.0$ and $\omega=0.1$ and these parametric values for particulate suspension are taken from the study of Apazidis [33]. The other parameters are set as: $N r=0.0,0.1,0.2, N_{A}=0.0,1.0,2.0$, $N_{B}=0.0,1.0,2.0, L n=L b=100.0, P e=R b=1.0, \alpha_{d}=0.1$ and $\Omega=0.1$. For verification, simulated results for skin friction coefficient and rate of heat transfer are compared with the published date and it is found that the solutions obtained by Siddiqa et al. [28], [32] can be recovered when nanofluid bioconvection is ignored. This comparison is appeared in Tab. 1 and results matches well with each other and shows good accuracy.

The computational data for skin friction coefficient, $\tau_{w}$, and rate of heat transfer, $Q_{w}$, for pure as well as contaminated water is presented in Tab. 2. It is noted from the second and third columns that the magnitude of the values of $\tau_{w}$ is minimized by increasing the values of mass concentration parameter, $D_{\rho}$, from 0.0 to 10.0. Specifically, this effect is enhanced when the value of buoyancy ratio parameter $N r$ is non-zero. While on contrary, last column of Tab. 2 shows that the rate of heat transfer, $Q_{w}$, is extensively promoted for non-zero values of $D_{\rho}$ and $N r$. Thus, the buoyancy ratio parameter together with the particle loading parameter can be used in boosting the rate of heat transfer and the physical reasons for such behavior of $D_{\rho}$ and $N r$ is given in later discussion of Figs. 1 and 2 .

Table 1: Comparison of $\tau_{w}$ and $Q_{w}$ with Refs. [28] and [32] for $D_{\rho}=0.0, \operatorname{Pr}=0.005$, $\omega=0.1$ and $\alpha_{d}=1.0$.

\begin{tabular}{|c|c|c|c|c|c|c|}
\hline \multirow{2}{*}{$X$} & \multicolumn{3}{|c|}{$\tau_{w}$} & \multicolumn{3}{c|}{$Q_{w}$} \\
\cline { 2 - 7 } & Present & Ref. [28] & Ref. [32] & Present & Ref. [28] & Ref. [32] \\
\hline 1.0 & 1.42742 & 1.41341 & 1.42524 & 0.04097 & 0.04170 & 0.05371 \\
3.0 & 1.42742 & 1.41341 & 1.42524 & 0.04097 & 0.04170 & 0.05371 \\
7.0 & 1.42742 & 1.41341 & 1.42524 & 0.04097 & 0.04170 & 0.05371 \\
9.0 & 1.42742 & 1.41341 & 1.42524 & 0.04097 & 0.04170 & 0.05371 \\
10.0 & 1.42742 & 1.41341 & 1.42524 & 0.04097 & 0.04170 & 0.05371 \\
\hline
\end{tabular}

Variation of buoyancy ratio parameter, $N r$, on skin friction coefficient $\tau_{w}$ and rate of heat transfer $Q_{w}$ is analyzed in Fig. 1. It can be noted from Eq. (28) that $N r$ acts as coupling factor for momentum equation and nanoparticle concentration equation. In order to make a comparison, $\tau_{w}$ and $Q_{w}$ are also plotted for $N r=0.0$. In principle, buoyancy effect the velocity and temperature gradients and thus it has a considerable influence on rate of heat transfer and wall skin friction coefficient. Fig. 1 illustrates that both $\tau_{w}$ and $Q_{w}$ reduces by increasing the values of $N r$ from 0.0 to 0.2 . Specifically, this effect is remarkable for skin friction coefficient. This may happens due to the reason that large values of buoyancy ratio parameter, $N r$, causes the base fluid to lose the thermal and kinetic energy from the inter-collision of dust particles and consequently both quantities tends to 
Table 2: Dimensionless wall skin friction and rate of heat transfer for $D_{\rho}=0.0,10.0, N r=$ 0.0,0.1 while $\operatorname{Pr}=7.0, \omega=\alpha_{d}=0.1, P e=R b=1.0, L b=L n=100.0, N_{A}=N_{B}=2.0$ and $\Omega=0.1$.

\begin{tabular}{|c|c|c|c|c|}
\hline \multirow{2}{*}{$X$} & \multicolumn{3}{|c|}{$\tau_{w}$} & \multicolumn{2}{c|}{$Q_{w}$} \\
\cline { 2 - 5 } & \multicolumn{4}{|c|}{$N r=0.0$} \\
\cline { 2 - 5 } & $D_{\rho}=0.0$ & $D_{\rho}=10.0$ & $D_{\rho}=0.0$ & $D_{\rho}=10.0$ \\
\hline 1.004 & 0.66520 & 0.53690 & 0.59707 & 0.97731 \\
2.003 & 0.66520 & 0.50119 & 0.59707 & 1.15878 \\
3.002 & 0.66520 & 0.47703 & 0.59707 & 1.29445 \\
5.000 & 0.66520 & 0.44379 & 0.59707 & 1.49751 \\
7.003 & 0.66520 & 0.42089 & 0.59707 & 1.65007 \\
9.001 & 0.66520 & 0.40381 & 0.59707 & 1.77254 \\
10.00 & 0.66520 & 0.39670 & 0.59707 & 1.82607 \\
\hline & \multicolumn{4}{|c|}{$N r=0.1$} \\
\hline 1.004 & 0.63998 & 0.50771 & 0.59258 & 0.97587 \\
2.003 & 0.63998 & 0.46979 & 0.59258 & 1.15888 \\
3.002 & 0.63998 & 0.44377 & 0.59258 & 1.29568 \\
5.000 & 0.63998 & 0.40739 & 0.59258 & 1.50036 \\
7.003 & 0.63998 & 0.38182 & 0.59258 & 1.65413 \\
9.001 & 0.63998 & 0.36238 & 0.59258 & 1.77757 \\
10.00 & 0.63998 & 0.35420 & 0.59258 & 1.83152 \\
\hline
\end{tabular}

reduce near the leading edge.

In order to see the influence of mass concentration of dust particle parameter, $D_{\rho}$, on the distribution of skin friction coefficient and rate of heat transfer, Fig. 2 is plotted. The numerical solutions of $\tau_{w}$ and $Q_{w}$ are discussed for pure as well as contaminated water. For $D_{\rho}=0.0$, this analysis recovers the solutions of classical problem of gyrotactic bioconvection of water based nanofluid. Here it is interesting to see that the plots for both of the physical quantities are effected sufficiently by the concentration of dust particles in bioconvection system. Fig. 2(a) reveals that the skin friction coefficient is very high for pure water and drastically reduces when $D_{\rho}$ changes from 0.0 to 10.0. The physical reason for such behavior can be attributed to the fact that, the carrier fluid loses the kinetic energy by loading the dust particles and this factor contributes in minimizing the velocity gradient at the wall and ultimately $\tau_{w}$ reduces. While on the other hand, an opposite behavior is recorded in the curves of rate of heat transfer coefficient, $Q_{w}$, in Fig. 2(b). Here, the water suspension gains the thermal energy due to the collision of particles, which increases the temperature gradient and eventually rate of heat transfer is promoted in boundary-layer regime.

Figure 3 is plotted to see the influence of combined effect of modified diffusivity ratio parameter, $N_{A}$, and the particle-density increment parameter, $N_{B}$, on $\tau_{w}$ and $Q_{w}$. It is observed from Fig. 3(a) that the skin friction coefficient reduces by magnifying the values of modified diffusivity ratio parameter $N_{A}$ from 0.0 to 2.0 when $N_{B}$ is taken as zero. But interestingly, in case of $\tau_{w}$ this behavior is reversed when the effect of particledensity increment parameter, $N_{B}$, is also taken into account. Fig. 3(a) further reveals that the skin friction is too high for large values of particle-density increment parameter, $N_{B}$. Thus, it can be concluded that the modified diffusivity ratio parameter, $N_{A}$, is more 
influential in promoting the skin friction coefficient together with the non-zero values of $N_{B}$. Furthermore, from Fig. 3(b) it is observed that the rate of heat transfer coefficient is maximum for $N_{A}=0$ and $N_{B}=0$. The plot of $Q_{w}$ remains insensitive by increasing the values of modified diffusivity ratio parameter, $N_{A}$, from 0.0 to 2.0 in the absence of particle-density increment parameter. But, the large values of $N_{A}$ acts as a retarding factor when the particle-density increment parameter, $N_{B}$, is penetrated into the the system. Therefore, the characteristics of heat transport of base fluid may notably be affected due to the suspension of small particles in heating fluids.

Contribution of modified diffusivity ratio parameter, $N_{A}$, and particle-density increment parameter, $N_{B}$, on velocity and temperature profiles for both phases is shown in Figs. 4 and 5, respectively. It can be seen from Fig. 4 that $U, U_{p}, \Theta$ and $\Theta_{p}$ remains almost invariant owing to an increase in the values of modified diffusivity ratio parameter. However, it is interesting to see that the velocity as well as temperature profiles for particle phase is always less than the corresponding profiles for fluid phase. This happens due to the presence of inert-particles in fluid, as they resist the flow and produce friction, which leads to a reduction in the magnitude of $U_{p}$ and $\Theta_{p}$. Thus, it can be concluded that $U_{p}$ and $\Theta_{p}$ decays quickly and attain their asymptotic behavior as compared to $U$ and $\Theta$. The effect of particle-density increment parameter, $N_{B}$, on velocity and temperature distribution is shown in Fig. 5. It can be seen that the non-zero values of $N_{B}$ are acting as a supporting driving force and accelerating the fluid flow, which ultimately promotes the velocity profiles for both phases, $U$ and $U_{p}$, in boundary layer region. The peaks of velocity curves in which non-zero values of $N_{B}$ are considered are relatively high, but it has no effect on $U$ and $U_{p}$ to attain their limiting behavior.

In order to show the variation of mass concentration parameter, $D_{\rho}$, and buoyancy ratio parameter, $N r$, on the plots of streamlines and isotherms, Figs. 6 and 7 are plotted. For the purpose of comparison, fluid suspension without dust particles, i.e., pure nanofluid, is also graphed in Fig. 6. As expectedly, by loading the dust particle in water based nanofluid, the velocity for particle phase reduces sufficiently as compared to the clear nanofluid case. This may happens due to the reason that particle clouds used to gather near the vicinity of vertical wall and offer resistance to fluid to flow, which results in reduction of velocity. Further, it is noted from Fig. 7 that the effect of $N r$ is more pronounced on the graph of streamlines and the physical reason for this influence is given in the earlier discussion of Fig. 1(a). The plots of isotherms in Fig. 7(b) reveals that the curves are almost insensitive by increasing the values of $N r$. Thus, non-zero values of buoyancy ratio parameter, $N r$, do not have notable influence on the distribution of temperature for water-particulate suspension.

\section{Conclusion}

The present study aims to present the numerical solutions of gyrotactic bioconvection boundary-layer flow of two-phase dusty fluid along an isothermally heated vertical surface. The major focus of this analysis is to visualize the flow characteristics of water based nanofluid when the solid dust particles are loaded into the mechanism. Primitive variable formulations (PVF) are applied to convert the governing equations of the carrier and the dispersed phase into another set of equations, which are then solved via two-point finite difference method together with tri-diagonal solver. Simulations are performed for water- 
based nanofluid containing the clouds of uniformly distributed dust particles. Effect of various emerging parameters are explored by expressing their relevance on rate of heat transfer, wall skin friction, velocity and temperature profiles, streamlines and isotherms. It is recorded that the buoyancy ratio parameter, $N r$, and mass concentration parameter, $D_{\rho}$, have notable influence in reducing the skin friction coefficient. It is also noted that the rate of heat transfer, $Q_{w}$, is extensively promoted owing to an increment in the values of $D_{\rho}$, whereas, a sufficient reduction is found in plots of $Q_{w}$ for non-zero values of particle-density increment parameter, $N_{B}$.

\section{References}

[1] Kuznetsov, A. V., Bio-thermal convection induced by two different species of microorganisms, Int. Commun. Heat Mass Transfer, 38, 2011, 548-553.

[2] Kuznetsov, A. V., The onset of thermo-bioconvection in a shallow fluid saturated porous layer heated from below in a suspension of oxytactic microorganisms, Eur. J. Mech. B/Fluids, 25, 2006, 223-233.

[3] Hill, N. A., Pedley, T. J., Bioconvection, Fluid Dyn. Res., 37, 2005, 1-20.

[4] Nield, D. A., Kuznetsov, A. V., The onset of bio-thermal convection in a suspension of gyrotactic microorganisms in a fluid layer: oscillatory convection, Int. J. Ther. Sci., 45, 2006, 990-997.

[5] Avramenko, A. A., Kuznetsov, A. V., Stability of a suspension of gyrotactic microorganisms in superimposed fluid and porous layers, Int. Commun. Heat Mass Transfer, 31, 2004, 1057-1066.

[6] Alloui, Z., Nguyen, T. H., Bilgen, E., Numerical investigation of thermo-bioconvection in a suspension of gravitactic microorganisms, Int. J. Heat Mass Transfer, 50, 2007, $1435-1441$.

[7] Spormann, A. M., Unusual swimming behaviour of a magneto tactic bacterium, FEMS Microbiol. Ecol., 45, 1987, 37-45.

[8] Pedley, T. J., Hill, N. A., Kessler, J. O., The growth of bioconvection patterns in a uniform suspension of gyrotactic microorganisms, J. Fluid Mech., 195, 1988, 223-237.

[9] Hill, N. A., Pedley, T. J., Kessler, J. O., Growth of bioconvection patterns in a suspension of gyrotactic microorganisms in a layer of finite depth, J. Fluid Mech., 208, 1989, 509-543.

[10] Sokolov, A., Goldstein, R. E., Feldchtein, F. I., Aranson, I. S., Enhanced mixing and spatial instability in concentrated bacterial suspensions, Phys. Rev. E., 80, 031903, 2009 .

[11] Tsai, T., Liou, D., Kuo, L., Chen, P., Rapid mixing between ferro-nanofluid and water in a semi-active Y-type micromixer, Sensors Actuators A Phys., 153, 2009, 267-273. 
[12] Kuznetsov, A. V., The onset of nanofluid bioconvection in a suspension containing both nanoparticles and gyrotactic microorganisms, Int. Commun. Heat Mass Transfer, 37, 2010, 1421-1425.

[13] Kuznetsov, A. V., Nanofluid bioconvection in water-based suspensions containing nanoparticles and oxytactic microorganisms: oscillatory instability, Nanoscale Research Letters, 6, 2011, 1-13.

[14] Kuznetsov, A. V., Non-oscillatory and oscillatory nanofluid bio-thermal convection in a horizontal layer of finite depth, Eur. J. Mech. B/Fluids, 30, 2011, 156-165.

[15] Buongiorno, J., Convective transport in nanofluids, ASME J. Heat Transfer, 128, 2006, 240-250.

[16] Kakaç, S., Pramuanjaroenkij, A., Review of convective heat transfer enhancement with nanofluids, Int. J. Heat Mass Transfer, 52, 2009, 3187-3196.

[17] Lee, J. H., Lee, S. H., Choi, C. J., Jang, S. P., Choi, S. U. S., A review of thermal conductivity data, mechanics and models for nanofluids, Int. J. Micro-Nano Scale Transp., 1, 2010, 269-322.

[18] Wong, K. F. V., Leon, O. D., Applications of nanofluids:current and future, Adv. Mech. Eng., 11, 2010, 519-659.

[19] Siddiqa, S., Hina, G., Begum, N., Hossain, M. A., Gorla, R. S. R., Numerical solutions of nanofluid bioconvection due to gyrotactic microorganisms along a vertical wavy cone, Int. J. Heat Mass Transfer, 101, 2016, 608-613.

[20] Aziz, A., Khan, W. A., Pop, I., Free convection boundary layer flow past a horizontal flat plate embedded in porous medium filled by nanofluid containing gyrotactic microorganisms, Int. J. Therm. Sci., 56, 2012, 48-57.

[21] Rudinger, G., Fundamentals of gas-particle flow, Elsevier Scientific Publishing Co., Amsterdam, 1980.

[22] Farbar, L., Morley, M. J., Heat transfer to flowing gas-solid mixtures in a circular tube, Ind. Eng. Chem., 49, 1957, 1143-1150.

[23] Marble, F. E., Dynamics of a gas containing small solid particles, combustion and propulsion, 5th AGARD colloquium, Pergamon press, 1963.

[24] Singleton, R. E., Fluid mechanics of gas-solid particle flow in boundary layers, Ph.D. Thesis, California Institute of Technology, 1964.

[25] Michael, D. H., Miller, D. A., Plane parallel flow of a dusty gas, Mathematica, 13, 1966, 97-109.

[26] Saffman, P. G., On the stability of laminar flow of a dusty gas, J. Fluid Mech., 13, 1962, 120-128.

[27] Michael, D. H., The steady motion of a sphere in a dusty gas,J. Fluid Mech., 31, 1968, 175-192. 
[28] Siddiqa, S., Hossain, M. A., Saha, S. C., Two-phase natural convection flow of a dusty fluid, Int. J. Numer. Method, 25, 2015, 1542-1556.

[29] Siddiqa, S., Begum, N., Hossain, M. A., Massarotti, N., Influence of thermal radiation on contaminated air and water flow past a vertical wavy frustum of a cone, Int. Commun. Heat Mass Transf., 76, 2016, 63-68.

[30] Siddiqa, S., Begum, N., Hossain, M. A., Compressible dusty gas along a vertical wavy surface, Appl. Math. Comput., 293, 2017, 600-610.

[31] Siddiqa, S., Begum, N., Hossain, M. A., Radiation effects from an isothermal vertical wavy cone with variable fluid properties, Appl. Math. Comput., 289, 2016, 149-158.

[32] Siddiqa, S., Begum, N., Hossain, M. A., Gorla, R. S. R., Numerical solutions of natural convection flow of a dusty nanofluid about a vertical wavy truncated Cone, Journal of Heat Transfer, 2017, DOI: 10.1115/1.4034815.

[33] Apazidis, N., Temperature distribution and heat transfer in a particle-fluid flow past a heated horizontal plate, Int. J. Multiphase Flow, 16, 1990, 495-513. 

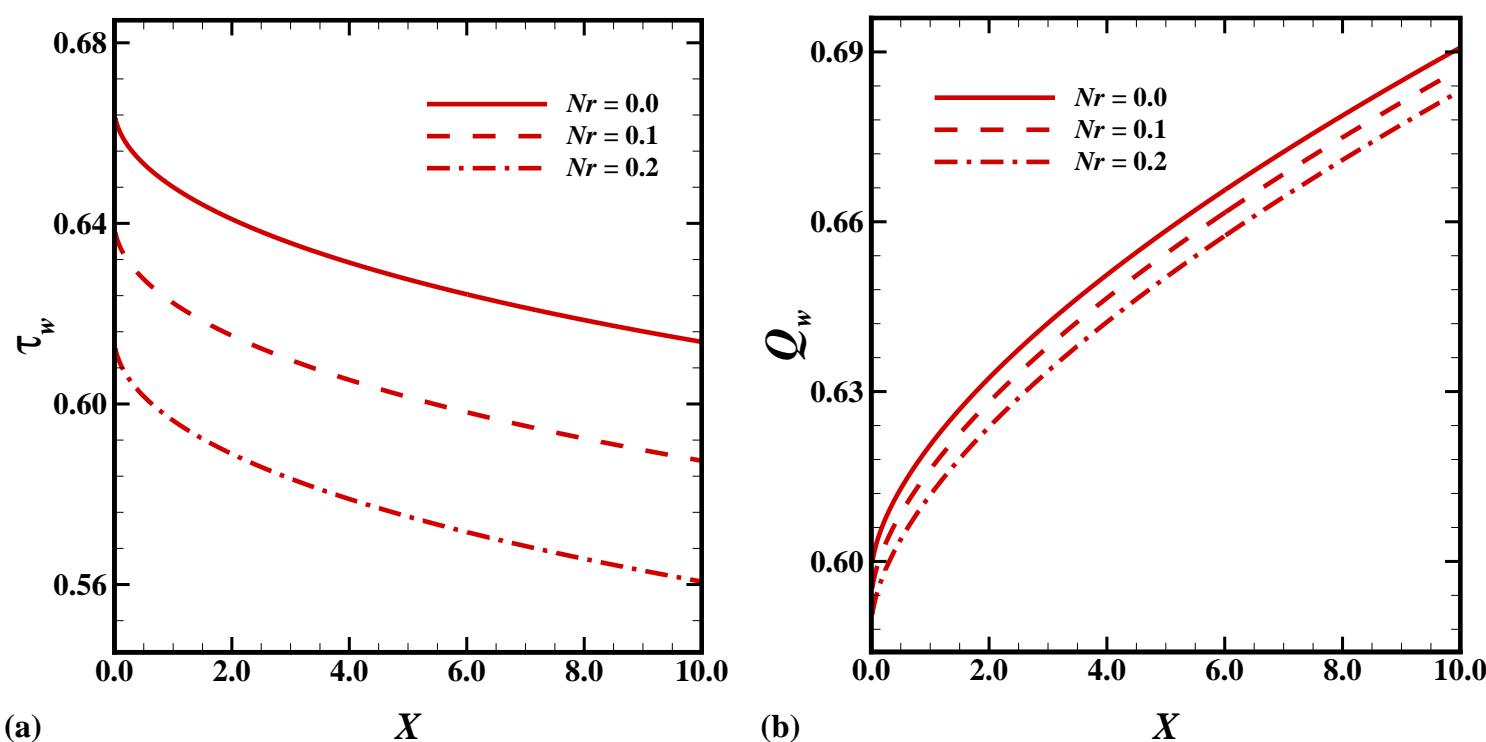

Fig. 1(a) Skin friction and (b) Rate of heat transfer coefficients for $N r=0.0,0.1,0.2$ while $\mathbf{P r}=7.0, \alpha_{d}=0.01, D_{\rho}=10.0, P e=1.0, L b=100.0$, $L n=100.0, R b=1.0, N_{A}=2.0, N_{B}=2.0, \omega=0.1$ and $\Omega=0.1$
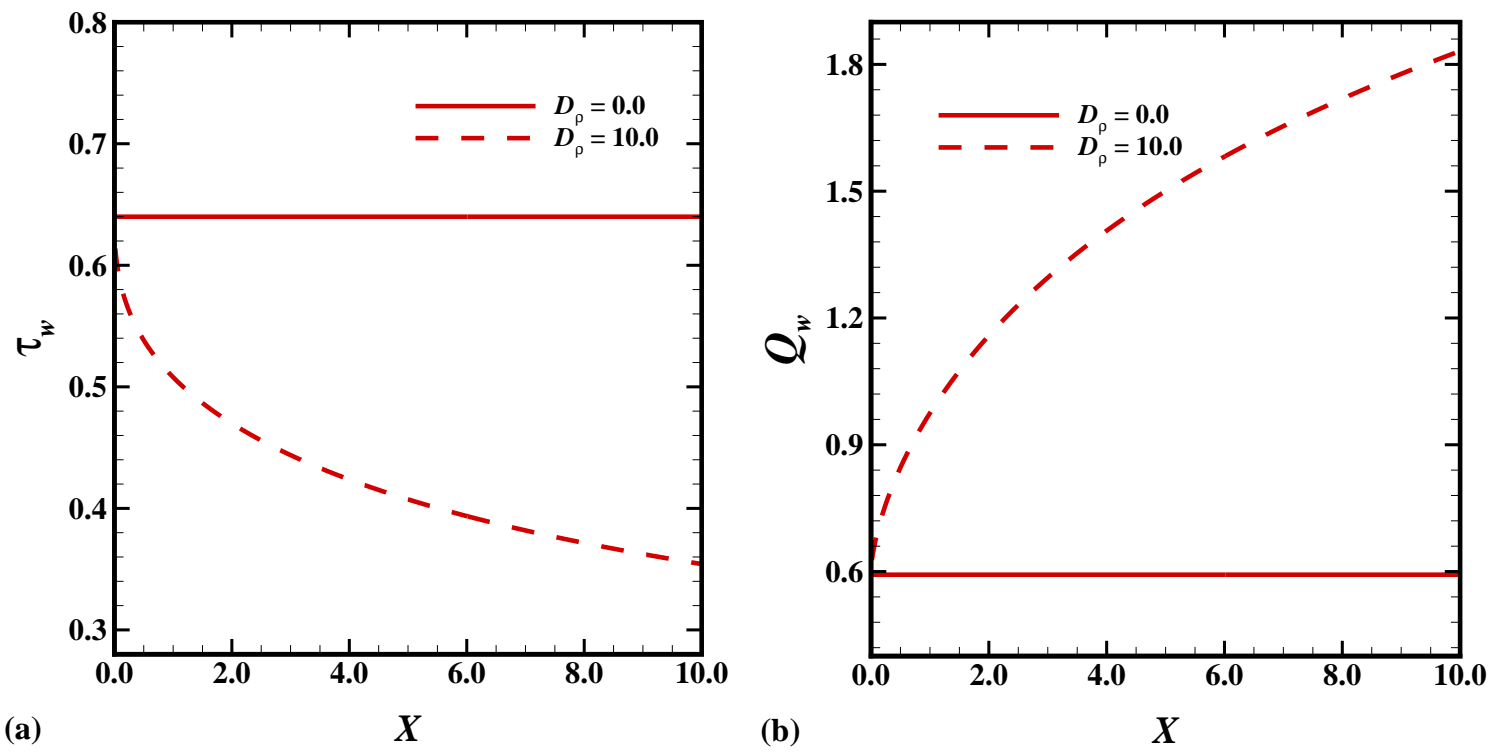

Fig. 2(a) Skin friction and (b) Rate of heat transfer coefficients for $D_{\rho}=0.0,10.0$ while $\mathbf{P r}=7.0, \alpha_{d}=0.1, N r=0.1, P e=1.0, L b=100.0, L n=100.0$, $R b=1.0, N_{A}=2.0, N_{B}=2.0, \omega=0.1$ and $\Omega=0.1$ 

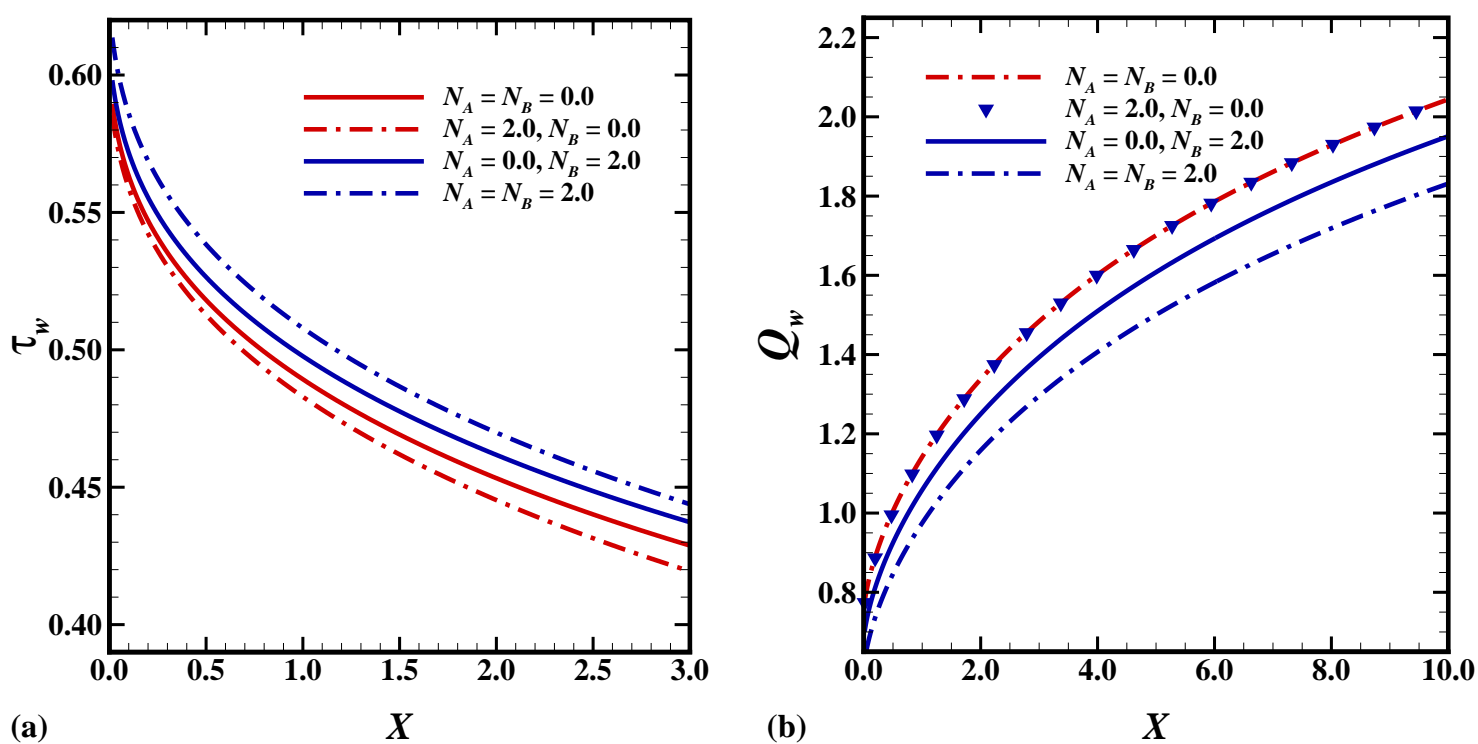

Fig. 3(a) Skin friction and (b) Rate of heat transfer coefficients for $N_{A}=0.0,2.0, N_{B}=0.0,2.0$ while $\mathbf{P r}=7.0, D_{\rho}=10.0, \alpha_{d}=0.1, N r=0.1, P e=1.0$, $L b=100.0, L n=100.0, R b=1.0, N_{A}=2.0, N_{B}=2.0, \omega=0.1$ and $\Omega=0.1$
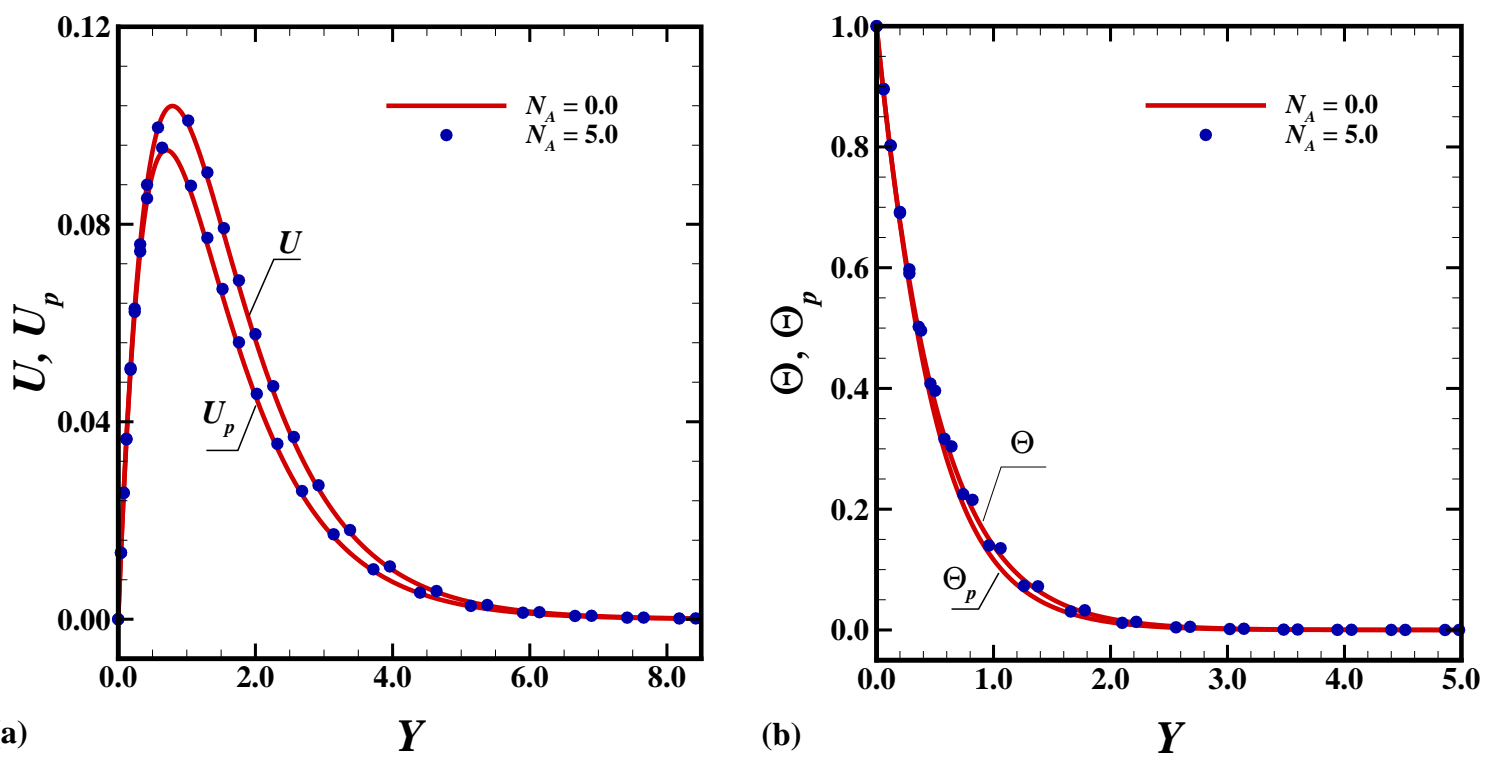

Fig. 4(a) Velocity and (b) Temperature profiles for $N_{A}=0.0,2.0$ while $\mathbf{P r}=7.0$, $D_{\rho}=10.0, \alpha_{d}=0.1, N r=0.1, P e=1.0, L b=100.0, L n=100.0, R b=1.0, N_{B}=2.0$, $\omega=0.1$ and $\Omega=0.1$ 

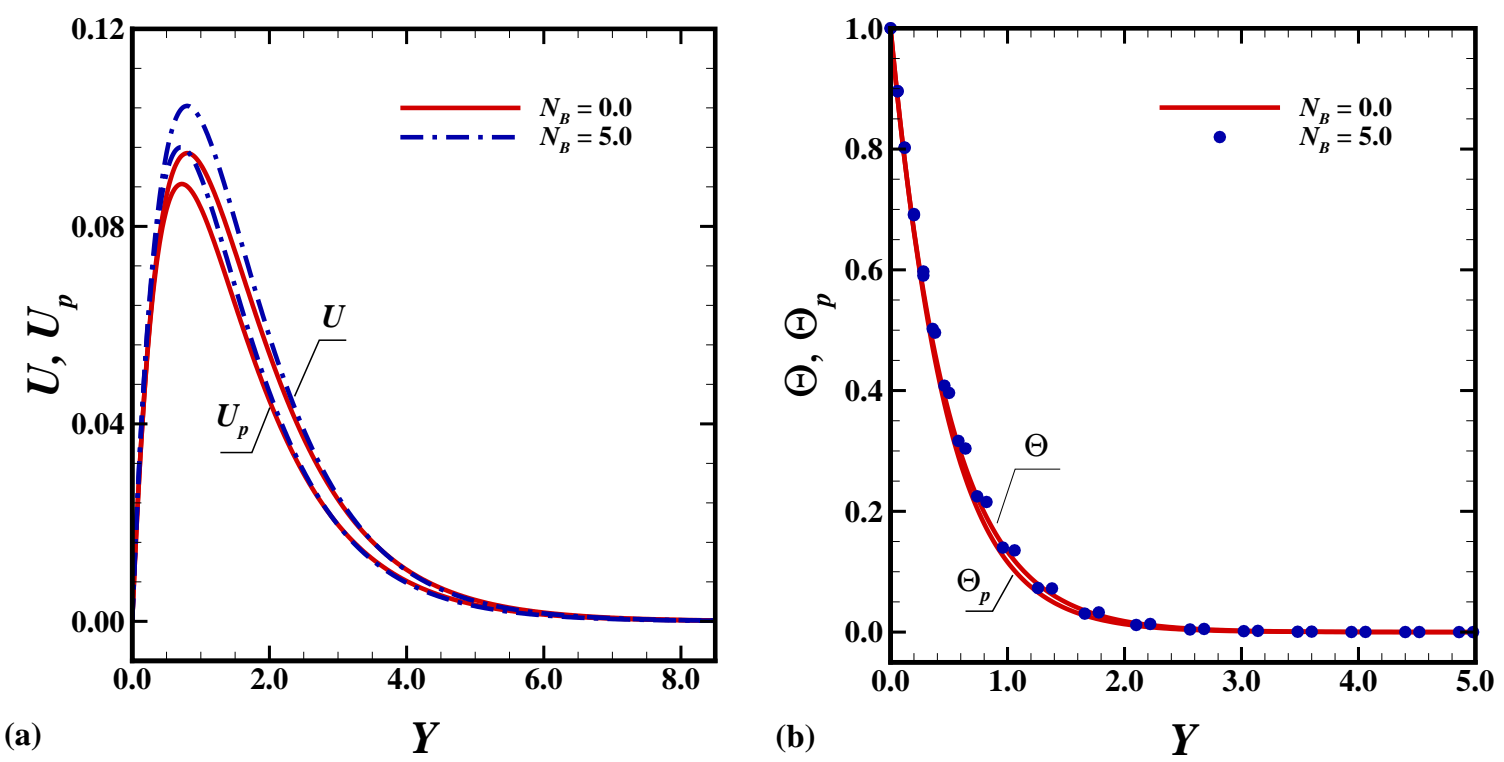

Fig. 5(a) Velocity and (b) Temperature profiles for $N_{B}=0.0,2.0$ while $\mathbf{P r}=7.0$, $D_{\rho}=10.0, \alpha_{d}=0.1, N r=0.1, P e=1.0, L b=100.0, L n=100.0, R b=1.0, N_{A}=2.0$, $\omega=0.1$ and $\Omega=0.1$
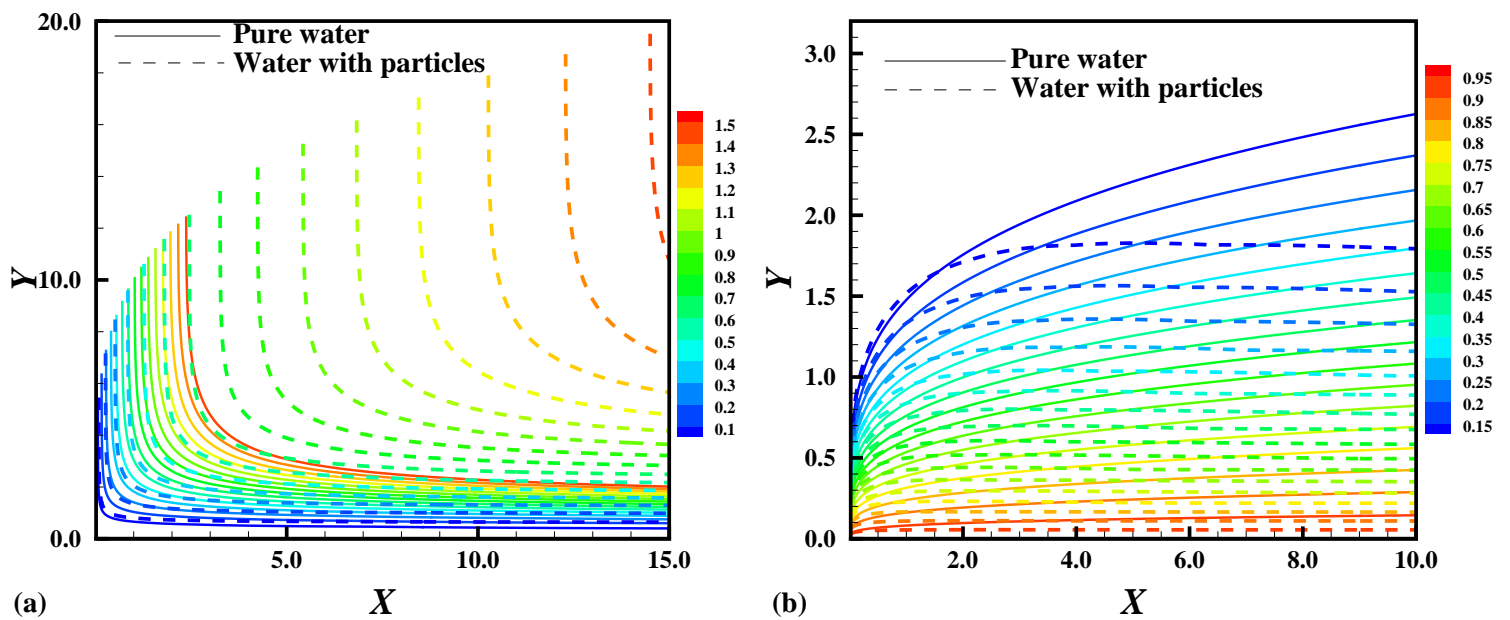

Fig. 6(a) Streamlines and (b) Isotherms for $D_{\rho}=0.0,10.0$ while $\mathbf{P r}=7.0$, $\alpha_{d}=0.1, N r=0.1, P e=1.0, L b=100.0, L n=100.0, R b=1.0, N_{A}=2.0, N_{B}=2.0$, $\omega=0.1$ and $\Omega=0.1$ 

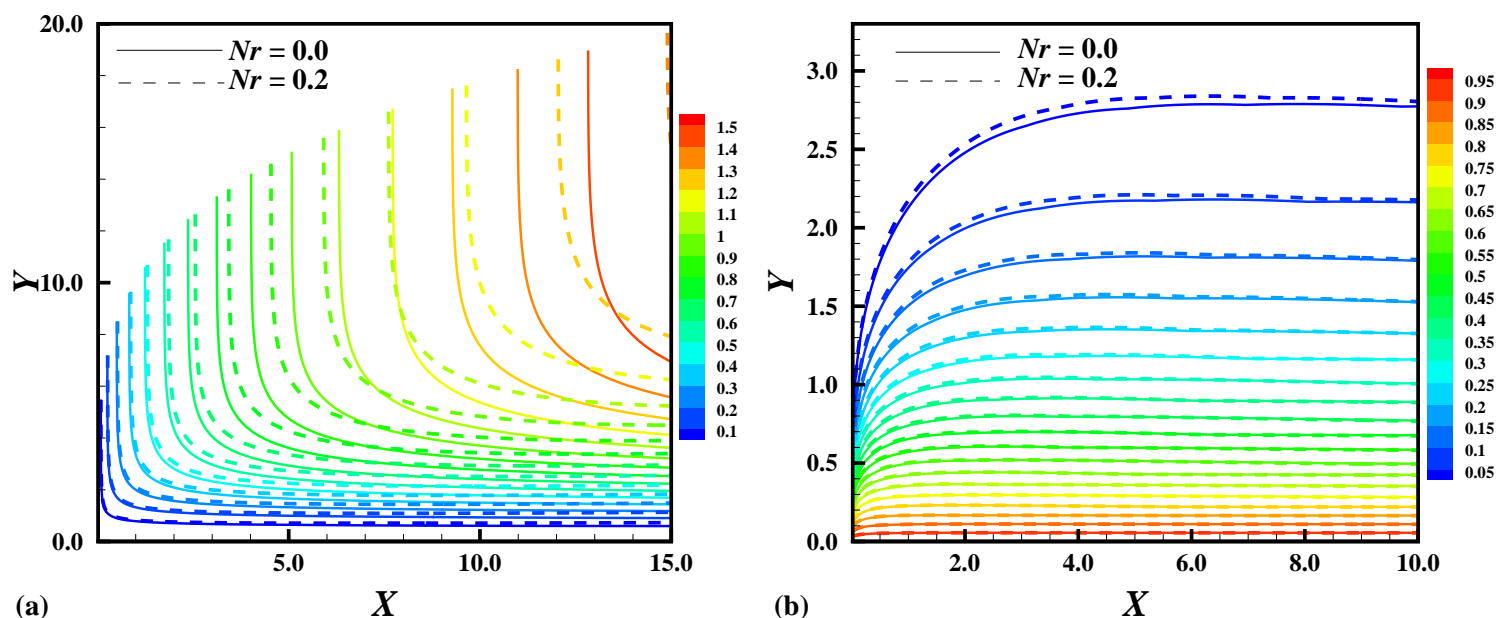

Fig. 7(a) Streamlines and (b) Isotherms for $N r=0.0,0.2$ while $\mathbf{P r}=7.0$, $\alpha_{d}=0.1, D_{\rho}=10.0, P e=1.0, L b=100.0, L n=100.0, R b=1.0, N_{A}=2.0, N_{B}=2.0$, $\omega=0.1$ and $\Omega=0.1$ 